\title{
Study on the Management System of English Teaching Expert Database Based on Computer Technology
}

\author{
Wang Ni \\ School of Humanities and International education, Xi'an Peihua University, Xi'an, Shaanxi, China \\ 619544785@qq.com
}

Keywords: computer technology; English teaching; expert database management system

\begin{abstract}
English education is a developing education systematic project. To a large extent, the difference of educational object's age, knowledge base and learning purpose determines the complexity of education. Therefore, the evaluation, selection and judgment of English education play an important role in the process of education. The construction of an English education expert selection system is of great significance and value for improving the performance of English education. Through the research, the English teaching of computer expert database management system should follow the principles: the principle of random replacement, odd principles; the main function of English teaching computer expert database management system includes user management module, expert management module, model management module; and put forward the expert hierarchical management model and expert system for dynamic management.
\end{abstract}

\section{Introduction}

English education is a developing education systematic project, and the complexity of education is largely determined by the differences of educational objects' age, knowledge base and learning purpose. Therefore, the evaluation, selection and judgment of English education play an important role in the process of education. ${ }^{[1]}$ The evaluation, selection and judgment are all made by men, so whether they are correct or not has a close relationship with decision makers' quality, experience and ability. The construction of an English education expert selection system is of great significance and value for improving the performance of English education. The experts have professional knowledge, judgment and experience, and they have the abilities to make recommendations and solve problems with these skills. The work of an expert is to provide knowledge for this system on how to perform tasks. The experts know which factors are important and understand the interrelationships among them. So far, there is no standard definition for an expert. However, the knowledge level and decision performance a person possesses are the key criteria for determining whether the person is an expert or not. Typical experts have some general features: first, their performance of solving problems is obviously better than that of ordinary people; and then, experts are relative; some may not be experts on other occasions. Therefore, it is of great significance and value to construct an expert selection system for English education in order to improve the performance of English education. ${ }^{[2]}$

\section{The Current Situation of the Expert Database Management System}

As for expert management systems, there is currently a lack of available and applicable methods and selection criteria. Generally, the reviewer chooses the experts who meet the basic requirements of the project to be evaluated according to the research direction, academic level and reputation of the experts, and they use the method of peer review to evaluate each other. Because of the objective reasons, the peer review method itself has some limitations, which exposes some shortcomings in the evaluation activities. In recent years, the academic circles both at home and abroad have doubts about the fairness of peer review method, believing that the academic level, academic attitude and moral quality of the evaluation experts will lead to the partiality of peer review. ${ }^{[3]}$ Establishing an 
English teaching expert database management system is of great significance to improve the quality of English teaching project evaluation. For the theoretical research and application practice of expert database management system, there have been some researches abroad. This system has been widely used in large academic search engines, such as SCI, SSCI, EI.

The evaluation of experts includes two parts. The first part is a comprehensive evaluation of the scientific research activities and achievements of an expert, which is a key and difficult problem in modern human resource management. It can provide important information for various kinds of human resource management decision- making, such as reward and punishment, promotion and selection. The other part is the performance evaluation which is closely related with the evaluation activities of science and technology projects. It is aimed at the work level and attitude of the experts in the evaluation activities, which is very important for the selection and adjustment of the evaluation experts. At present, there are many problems to be solved in the research of expert selection system theoretically and practically, such as the representativeness and clarity of the indicators of evaluation system, the rigor of the indicator level, the quantitative evaluation of indicators, the indicators' weight selection method, the decision makers' weight selection method, the selection of evaluation decision making models, the process control of evaluation decision making, and so on. Besides, because of lacking evaluation credit in the construction of expert database, experts are selected subjectively only according to the information they provided, which makes it difficult to guarantee the quality of the experts.

\section{The Structure of the Management System of English Teaching Expert Database}

The expert selection system is simply a system that stores expert information, selects appropriate experts for specific educational content and manages experts according to the results of evaluation. In general, when a person is admitted to an expert database, he is located into corresponding level according to the indicators of professional field, technical title, working life and so on. After selecting the expert for a project evaluation, it is necessary to assess the expert's practical skills and judge the expert's performance level to determine the priority of his subsequent selection. Finally, if the performance of the expert has always been low, and the academic level, reputation have not changed much, it is necessary to adjust the expert hierarchy or delete him from the expert database.

\section{The Principles of Expert Selection for the Computer Expert Database Management System}

The English teaching computer expert database management system should follow the odd number principle, random principle and replacement principle.

\subsection{The Odd Number Principle}

When selecting experts for evaluation, the number of experts should be odd, which could facilitate managers to make decisions when experts holding opposite views.

\subsection{The Random Principle}

The evaluation expert group or committee participating in collective evaluation activities should be selected randomly from the evaluation expert database according to the requirements. They should be the representativeness of different disciplines, different professional techniques, different units and regions, and there should be a certain proportion of experts engaged in practical education work.

\subsection{The Replacement principle}

In the process of evaluating specific educational and teaching projects, experts should be periodical replaced in different projects 


\section{The Main Functional Modules of the Computer Expert Database Management System}

Scientific division of application module is the basis of computer expert management system serving English education. The main functional modules of computer expert database management system for English teaching include user management, expert management, model management and so on.

\subsection{The user management}

The users of this system are divided into three types: the first one is the system administrator, who has all the permissions of the system and can operate on all modules; the second one is the general manager, who has the access to all modules except operating on the expert database as well as adjusting ranks of experts; the last one is the general user, who can query the information of experts and their business and select proper experts. ${ }^{[4]}$

\subsection{The expert management}

The expert management contains adding, modifying, deleting and querying the information of experts. In addition to some basic information, it also includes experts' information on their teaching performance, scientific researches, papers, works, awards, specialization, the years of working in professional field, their levels and related English teaching programs. Based on the various information of experts and evaluation mathematical model, the system can calculate the scores of the basic evaluation of experts and the degree of project correlation and sort the experts according to the magnitude of the scores. The classification criteria are formulated and the experts are classified into four layers, including top experts, optimum experts, experts and candidate experts.

\subsection{The model management}

The model management is divided into two parts: one is the management of expert evaluation index and index weight; the other one is the management of comprehensive evaluation model. The comprehensive evaluation model usually includes grey multi-hierarchal synthetic evaluation model and mathematical statistical analysis model. The grey multi-hierarchal synthetic evaluation model can be implemented by internal programming of expert database management system, while the mathematical statistical analysis model and the intelligent evaluation model can be implemented in the form of correlation analysis module integrating SPSS and MATLAB software.

\section{The Expert Hierarchy in the Computer Expert Database management system}

\subsection{The classification standard of the expert hierarchy}

Based on the evaluation results of basic evaluation and performance evaluation, experts are divided into four layers, including top experts, optimum experts, experts and candidate experts. Top experts are those who enjoy outstanding achievements and professional prestige in the field of English education, accounting for a small part of the total number of the expert database. The optimum experts and experts account for the majority of the total number, referring to those who scored higher on basic assessment and performed well in specific English education projects. They are also more likely to be selected to participate in the actual business. The candidate experts refer to those who scored poorly in basic evaluation and performance evaluation and are less likely to be selected to participate in the actual business.

\subsection{The dynamic mechanism of the expert hierarchy}

In order to maintain the reasonable structure of expert hierarchy and the dynamic mechanism of eliminating and optimizing, the corresponding proportional of top and optimum experts should be $20 \%$, the general experts $60 \%$ and candidate experts $20 \%$. The specific control proportion is based on the overall level of the experts in the database at this stage, and can be flexibly adjusted by the manager of the expert database. 


\section{Expert Correlation Calculation of the Computer Expert Database Management System}

The evaluation of the correlation degree of English teaching is to reasonably identify the relevance degree between experts and a specific English teaching project. The result of the evaluation is an important supplement to the basic evaluation and comprehensive evaluation in the expert selection system. The objective of correlation degree evaluation is to establish the relationship between the evaluation object and the expert attribute and to quantify it. Correlation degree and comprehensive evaluation are evaluation indicators at the same level. Correlation degree should be regarded as a strong key word in order to ensure that the corresponding experts in the English teaching projects rank first. At the same time, it should be noted that the ranking of relevance degree is dynamic. As for different English teaching programs, the corresponding relevance degree ranking of experts in English teaching is different. Based on the information of experts' English teaching areas, corresponding positions and years of experience, the idea of correlation evaluation is used to calculate the correlation degree between experts and specific English teaching projects.

\section{Expert Dynamic Management of the Computer Expert Database Management System}

In the initial stage of the establishment of the English teaching expert database, the experts are divided into different levels according to the basic evaluation index system and the proportion of the four levels of experts, and the segmentation points will be formed at the same time. With the use of the system and the passage of time, there will be an expert's supplement. For the subsequent experts, they will be classified into different levels according to the basic information evaluation index and the corresponding hierarchical segmentation points. ${ }^{[5]}$

With the addition of expert information, the segmentation points should be adjusted accordingly. After the system is used for a certain period (the period should be longer than the period of information change and expert supplement), the basic information of experts in the database should be re-evaluated and the hierarchical segmentation points should be revised according to the hierarchical control ratio. On the one hand, the dynamic management of basic evaluation can track the change of key information of expert's basic information, adjusting its current level according to the changes; on the other hand, it can master the overall level of the experts' basic ability in the database, accumulating experience for hierarchical division and the hierarchical segmentation points can be used to classify the new experts into corresponding levels.

\section{Expert Decision-Making Process of the Computer Expert Database Management System}

In the English teaching computer expert database management system, the following steps and methods can be taken in the process of participating in the teaching program or teaching plan design. First, the panel of experts should be formed according to the scope of knowledge and future application areas, no more than 20. Second, the expert responsible for the project will explain to the members the general situation of the project and the background information and application scope of the construction of index system as well as its objects, enabling the experts to have a certain understanding of the purpose and characteristics of the index system. Third, the questions and relevant requirements for the demonstration of the index system should be put forward to all experts, attaching all the background materials on this issue, i.e. the indicator system and its construction instructions, and the experts should make written answers. Fourth, experts present their opinions on the basis of the materials they receive, and explain how they use them and ask questions. Fifth, the opinions of all experts are gathered, summarized and distributed to the experts. The collection of opinions and information feedback is conducted in three rounds, and the opinions given by experts are anonymous. The last, the experts' opinions should be dealt with comprehensively and discussed at the meeting. After several rounds of information feedback, experts will have a deeper understanding of the index system. On this basis, the final opinions will be made public and the problems existing in the index system will be demonstrated through the method of collective 
discussion of experts, and the optimization method of the index will be finally determined.

\section{Conclusion}

The theory and method of decision support system play an important role in the economic and social life at the individual level, enterprise level and even national level. Establishing a computer expert database management system for English teaching is a concrete application of the theory and method of decision support system in the field of education. It is of great theoretical and practical significance for improving English teaching performance with the external intelligence resources of English Education. In the process of follow-up research and implementation, we should strengthen the organizational leadership and guarantee mechanism for the application of computer expert database management system in English teaching, which will be the focus of follow-up researches.

\section{References}

[1] Liu Haitao. Practice and thinking on the credit transfer system in the colleges and universities in Europe and North America-based on the analysis of ECTS in Europe and CAA in North Carolina, US[J]. Studies in Foreign Education, 2016 (12): 106-115

[2] Lin Shiyuan, Chen Li and Peng Yiping. Towards an external quality assurance system for distance education provision of Chinese universities[J]. Distance Education in China, 2016 (05): 43-49

[3] Li Xiuli. Study on the application skills of business English translation in international trade[J]. Overseas English, 2014 (5): 179-180

[4] Tian jinchang and Li xiaoling. The innovation and development of college teacher appraisal and management mechanism based on the competent capability theory[J]. Journal of Shaanxi Institute of Education, 2009 (12): 88-91

[5] Chen zhimin, Lv jinqiao and Liu Meifeng. Microlesson instructional design and the analysis of its common problems- the analysis of 295 works from "The 13th National Multimedia Courseware Competition” [J]. Modern Educational Technology, 2014 (8): 20-27 